\title{
Pediatric clinical practice guidelines in China: still a long way to go
}

\author{
Jie Chen ${ }^{1}[$
}

Received: 14 September 2018 / Accepted: 20 September 2018 / Published online: 29 September 2018

(c) Children's Hospital, Zhejiang University School of Medicine 2018

Clinical practice guidelines provide optimal patient care recommendations, which are important tools for promoting the equality of health care services and saving healthcare resources in China [1]. The evidence-based clinical guidelines are specific to clinical problems and are developed through systematic reviewing to help clinicians and patients make appropriate decisions. Chen et al. revealed several limitations in adoption and adaptation of Chinese Guidelines including (1) rarely using systematic review to support recommendations, (2) lacking information in conflict of interest, and (3) low adherence rate of clinicians to guidelines [1]. The overall quality of China's clinical guidelines still needs improvement compared with the European and American guidelines. China currently lacks capacity for evidence-based guideline development and coordination by a central agency. Most Chinese guideline users rely on recommendations developed by professional groups that lack demonstration of transparency, including conflict of interest management and evidence synthesis, and quality. Taking into account the development of the guidelines at the national level, the Standards Division of the National Health Commission of China commissioned the Evidence-Based Medical Center of Fudan University to develop China's guideline evaluation criteria in 2017. Chinese experts are expected to familiarize themselves with the rules for developing guidelines, and use evidence-based medicine methods to standardize the development of high-quality guidelines. I think that the quality of the clinical guidelines will be improved in the near future.

The English version of the guidelines not only shows the world the epidemiological data, medical evidence and diagnosis and treatment models with Chinese characteristics, but also plays an important role in promoting international dialogue and expanding academic influences on a global scale.

Jie Chen

hzcjie@zju.edu.cn

1 Department of Gastroenterology, Children's Hospital, Zhejiang University School of Medicine, Hangzhou 310051, China
In the current issue of World Journal of Pediatrics, we published English version of three pediatric clinical practice guidelines in China including assessment and provision of nutrition support therapy in critically ill children [2], management of acute infectious diarrhea in Chinese children [3], and diagnosis and treatment of hand, foot and mouth disease [4].

Nutritional support therapy has been widely recognized as an important part of the overall treatment of critically ill children, but it often faces difficulties and challenges in implementation. Chinese guidelines for the assessment and provision of nutrition support therapy in critically ill children is formulated by Group of Chinese Pediatrics Society, Pediatrics Group of Chinese Emergency Society (both afflicted to Chinese Medical Association), and Developing and Appraising Center of Pediatric Clinical Practice Guidelines of Children's Hospital, Fudan University. The guidelines emphasized the importance of nutritional assessment, particularly the detection of malnourished patients. The target population of the guidelines is critically ill patients aged from 1 month to 18 years and length of stay greater than 2 or 3 days in a PICU [2].

Infectious diarrhea is a global infectious disease with a high incidence and widespread epidemics, which is a serious public health challenge to humans, especially to children. In China, according to the household survey data of some provinces, the overall incidence of diarrhea is $0.17-0.70$ episodes per person-year, and that for children under 5 years is 2.50-3.38 episodes per person-year. Chinese practice guidelines for acute infectious diarrhea in children was developed by the an expert working group composed of pediatric gastroenterology, pediatric infectious disease and epidemiology experts under the organization of Academic Group of Pediatric Gastroenterology of Chinese Pediatrics Association. The working group put forward clinical problems for most controversial treatments, including the fluid selection in rehydration of severe dehydration due to acute diarrhea, lactose-free diet, antibiotics and non-specific anti-diarrheal drugs, and search the literatures for the proposed clinical problems, collect evidence, apply evidence-based methods 
for evidence evaluation to form preliminary recommendations, and then use open-ended discussions to solicit expert opinions to form recommendations [3].

For the epidemic situation of hand, foot and mouth disease, China has issued three versions of guiding medical treatment guidelines or expert consensus since 2010, which played a positive role in guiding medical institutions to diagnose and treat hand, foot and mouth disease scientifically, especially for severe hand, foot and mouth disease. The early identification and treatment of severe hand, foot and mouth disease has been improved. However, the incidence of hand, foot and mouth disease is still high. The pathogen spectrum has changed, and the clinical manifestations caused by different pathogens are different. To manage hand, foot and mouth disease more effectively, and to reduce the morbidity and mortality of severe hand, foot and mouth disease, the National Health Committee organized specialists in related fields including infectious disease, disease control, pediatrics, hospital sensation and consults to develop the Hand, Foot and Mouth Disease Diagnosis and Treatment Guide (2018 Edition) based on analysis of the latest domestic and foreign literature and integration of clinic experience. The guideline summarizes the epidemic changes of hand, foot and mouth disease and the progress of diagnosis and treatment in recent years. It is the guiding plan for the diagnosis and treatment of hand, foot and mouth disease [4].

Although pediatric guidelines are not a panacea for all health problems in children in China, there is no doubt that the development and implementation of high-quality evidence-based guidelines in line with national practice improve healthcare in Chinese pediatric patients. The pediatric clinical practice varies widely among different regions and hospitals. The pediatric guidelines are particularly important for the development of pediatric healthcare and improvement of medical equality throughout China. China has about 250 billion children, and accounting for $15 \%$ of the children worldwide. Since the widely adoption of "Two-Child Policy" in China, the current pediatric healthcare resources is far behind the increasing demand of pediatric healthcare delivery. The gradually increased number of births per year exerts great pressure on the insufficient pediatric healthcare resources. Therefore, high-quality and applicable pediatric clinical practice guidelines are imperatively important for this severe situation of pediatric healthcare delivery in China. The guidelines adopt cost-effective medical interventions and healthcare strategies that will help optimize the use of current pediatric healthcare resources.

In summary, China has a large population base of children, and there are a variable of diseases remained to be studied. The Healthy China 2030 Plan proposed by the Chinese government has strengthened the support and the quality requirements for clinical researches [5]. Thus, a large number of high-quality evidences from Chinese clinical researches may be written into the guidelines in the near future. The quality, promotion and application of the pediatric clinical guidelines developed in China will also be improved, though with a long way to go.

Author contributions CJ drafted and revised the manuscript.

Funding No funding for this manuscript.

\section{Compliance with ethical standards}

Ethical approval Not needed.

Conflict of interest No financial or nonfinancial benefits have been received or will be received from any party related directly or indirectly to the subject of this article.

\section{References}

1. Chen YL, Wang C, Shang HC, Yang KH, Norris SL. Clinical practice guidelines in China. BMJ. 2018;360:j5158.

2. Zhu XM, Qian SY, Lu GP, Xu F, Wang Y, Liu CF, et al. Chinese guidelines for the assessment and provision of nutrition support therapy in critically ill children. World J Pediatr. 2018;14:419-28.

3. Chen J, Wan CM, Gong ST, Fang F, Sun M, Qian Y, et al. Chinese clinical practice guidelines for acute infectious diarrhea in children. World J Pediatr. 2018;14:429-36.

4. Li XW, Ni X, Qian SY, Wang Q, Jiang RM, Xu WB, et al. Chinese guidelines for the diagnosis and treatment of hand, foot and mouth disease (2018 edition). World J Pediatr. 2018;14:437-47.

5. The Healthy China 2030 Plan. http://www.mohrss.gov.cn/SYrlz yhshbzb/zwgk/ghcw/ghjh/201612/t20161230_263500.html. Accessed 15 Aug 2018. 